\title{
Compact Finite Difference Scheme Combined with Richardson Extrapolation for Fisher's Equation
}

\author{
Hailu Muleta Chemeda (iD, Ayana Deressa Negassa ${ }^{(D,}$, and Feyisa Edosa Merga \\ Jimma University Department of Mathematics, Jimma, Ethiopia \\ Correspondence should be addressed to Hailu Muleta Chemeda; hailux2020@gmail.com
}

Received 7 December 2021; Accepted 23 December 2021; Published 27 January 2022

Academic Editor: Francisco Urena

Copyright ( $) 2022$ Hailu Muleta Chemeda et al. This is an open access article distributed under the Creative Commons Attribution License, which permits unrestricted use, distribution, and reproduction in any medium, provided the original work is properly cited.

In this study, the fourth-order compact finite difference scheme combined with Richardson extrapolation for solving the 1D Fisher's equation is presented. First, the derivative involving the space variable is discretized by the fourth-order compact finite difference method. Then, the nonlinear term is linearized by the lagging method, and the derivative involving the temporal variable is discretized by the Crank-Nicolson scheme. The method is found to be unconditionally stable and fourth-order accurate in the direction of the space variable and second-order accurate in the direction of the temporal variable. When combined with the Richardson extrapolation, the order of the method is improved from fourth to sixth-order accurate in the direction of the space variable. The numerical results displayed in figures and tables show that the proposed method is efficient, accurate, and a good candidate for solving the 1D Fisher's equation.

\section{Introduction}

Mathematical modeling of most physical systems leads to linear/nonlinear partial differential equations (PDEs) in various fields of science. PDEs have enormous applications compared to ordinary differential equations (ODEs) such as in dynamics, electricity, heat transfer, electromagnetic theory, quantum mechanics, and so on [1].

The 1D Fisher's equation is given by

$\frac{\partial u}{\partial t}=\beta \frac{\partial^{2} u}{\partial x^{2}}+\alpha u(1-u), \quad x \in(a, b) \subseteq(-\infty, \infty), t \geq 0$.

With the initial condition,

$$
u(x, 0)=f(x), \quad a \leq x \leq b .
$$

The boundary conditions are

$$
\begin{aligned}
& u(a, t)=g_{1}(t), \\
& u(b, t)=g_{2}(t), \quad t \geq 0,
\end{aligned}
$$

where $\beta(>0)$ is the diffusion coefficient, $\alpha(>0)$ is the reactive factor, $x$ is the distance, $t$ is the time, and $u(x, t)$ is the population density. $f(x), g_{1}(t)$, and $g_{2}(t)$ are the smooth functions on the given domain.

The 1D Fisher's equation in equation (1) was first proposed by Fisher [2] as a model for the spatial and temporal propagation of a virile gene in an infinite medium [3]. It can also be considered as a model equation for the evolution of a neutron population in a nuclear reactor $[4,5]$. Equation (1) also describes the rate of the advance of a new advantageous gene within a population of a constant density occupying a one-dimensional habitat [6]. In equation (1), the effect of the linear diffusion is observed along $u_{x x}$, whereas the nonlinear local multiplication or reaction is observed along $u(1-u)[5,7,8]$. Some of the application areas of equation (1) include gene propagation $[3,5]$, tissue engineering [9], combustion [10], and neurophysiology [8].

Due to its wider applications in the real world problems, many researchers have been developing both analytical and numerical methods for solving Fisher's equation. Gazdag 
and Canosa [6] applied accurate space derivatives (ASD) which can be carried out efficiently by the use of the fast Fourier transformation algorithm to the numerical solution of equation (1). The Petrov-Galerkin finite element method [11], Sinc collocation method [12], and the wavelet Galerkin method [13] have been applied for solving equation (1). Bastani and Salkuyeh [14] studied the numerical solution of equation (1) using the compact difference method (FDM). Hasnain et al. in [5] also studied equation (1) numerically using the Crank-Nicolson scheme combined with the Richardson extrapolation technique.

A widely and frequently used numerical technique for Fisher's equation is the FDM. However, the usual FDM shows shortcomings in computational accuracy. The two widely known methods used to improve such shortcomings are the application of the compact finite difference method and Richardson extrapolation technique [15].

Due to its high accuracy and stability property, the compact finite difference method attracts much attention from many scholars for finding approximate solutions of various kinds of equations [16], and it is much more accurate than the corresponding explicit scheme of the same order [17]. A highorder compact finite difference method was applied for systems of reaction-diffusion equations in [18], and the Helmholtz equation was approximated by a sixth-order compact finite difference (CFD6) method in [19]. Dennis et al. [20] proposed the fourth-order CFDS for convection-diffusion problems. Bastani and Salkuyeh [14] had combined a CFD6 scheme for second derivative in space and a third-order total variation diminishing the Runge-Kutta (TVD-RK3) scheme in time to approximate Fisher's equation.

Another way for improving the accuracy and rate of convergence of the FDM is through the application of Richardson's extrapolation (RE) provided that their error term is expressible as a polynomial or power series in $h$ $[21,22]$. Furthermore, RE does not require any knowledge of the underlying methodology except the order of accuracy, which guarantees the minimal intervention to the existing computational tools [23]. Gordin [24] applied the RE method to improve a fourth-order CFDS to sixth-order in 1D parabolic equations and Schrödinger-type equations. Compared to low-order methods, high-order methods can achieve satisfactory errors on much coarser grids and thus greatly curtail the computational cost [25].

Although mathematical properties of Fisher's equation and plenty of discussion are available in the literature, majority of them do not address the import properties such as stability analysis, order of convergence, and consistence of the underlying numerical method. The aim of this study is to develop a higher-order numerical method for solving the $1 \mathrm{D}$ Fisher's equation. We also establish the stability condition and order of convergence of the proposed method.

\section{Mathematical Formulation and Analysis of the Proposed Method}

Let the spatial solution domain $[a, b]$ in equation (1) be divided into $N$ equal subintervals with uniform mesh size $h$ which can be represented as $h=(b-a) / N$, such that $x_{i}=a+i h$ for $i=0,1,2, \ldots, N$. Similarly, the temporal solution domain $[0, T]$ can also be divided into $M$ equal subintervals with uniform width $k=T / M$, such that $t_{j}=j k$ for $j=0,1,2, \ldots, M$.

To linearize the nonlinear term in equation (1), the method of lagging [26] is used in such a way one of the nonlinear term is approximated at $(j+1)^{\text {th }}$ time level and the other is approximated at $j^{\text {th }}$ time level.

2.1. Fourth-Order Compact Scheme. From Taylor's series expansion, the first and second derivatives of $u$ can be approximated as follows:

$$
\begin{aligned}
& u_{i}^{\prime}=\delta_{x} u_{i}-\frac{h^{2}}{6} u_{i}^{(3)}-\frac{h^{4}}{120} u_{i}^{(5)}+O\left(h^{6}\right), \\
& u_{i}^{\prime \prime}=\delta_{x}^{2} u_{i}-\frac{h^{2}}{12} u_{i}^{(4)}-\frac{h^{4}}{360} u_{i}^{(6)}+O\left(h^{6}\right) .
\end{aligned}
$$

Here,

$$
\begin{aligned}
\delta_{x} u_{i} & =\frac{u_{i+1}-u_{i-1}}{2 h}, \\
\delta_{x}^{2} u_{i} & =\frac{u_{i+1}-2 u_{i}+u_{i-1}}{h^{2}} .
\end{aligned}
$$

Substituting equation (5) into (1) yields

$$
\frac{\partial u_{i}}{\partial t}=\beta\left(\delta_{x}^{2} u_{i}-\frac{h^{2}}{12} u_{i}^{(4)}\right)+\alpha u_{i}\left(1-u_{i}\right) .
$$

Differentiating equation (1) twice with respect to the space variable and rearranging, we obtain

$$
\begin{aligned}
\frac{\partial^{4} u_{i}}{\partial x^{4}}= & \frac{1}{\beta}\left(\frac{\partial^{2}}{\partial x^{2}}\left(\frac{\partial u_{i}}{\partial t}\right)\right) \\
& +2 \frac{\alpha}{\beta}\left(\frac{\partial u_{i}}{\partial x}\right)^{2}-\frac{\alpha}{\beta}\left(1-2 u_{i}\right) \frac{\partial^{2} u_{i}}{\partial x^{2}} .
\end{aligned}
$$

Replacing the first and second derivatives of $u$ in equation (8) by equations (4) and (5), respectively, we get

$$
\begin{aligned}
\frac{\partial^{4} u_{i}}{\partial x^{4}}= & \frac{1}{\beta}\left(\frac{\partial^{2}}{\partial x^{2}}\left(\frac{\partial u_{i}}{\partial t}\right)\right) \\
& +2 \frac{\alpha}{\beta}\left(\delta_{x} u_{i}\right)^{2}-\frac{\alpha}{\beta}\left(1-2 u_{i}\right) \delta_{x}^{2} u_{i} .
\end{aligned}
$$

Now, substituting equation (9) into (7) and rearranging yields

$$
\begin{aligned}
\left(1+\frac{h^{2}}{12} \delta_{x}^{2}\right) \frac{\partial u_{i}}{\partial t}= & \beta \delta_{x}^{2} u_{i}-\frac{\alpha h^{2}}{6}\left(\delta_{x} u_{i}\right)^{2} \\
& -\frac{\alpha h^{2}}{12}\left(1-2 u_{i}\right) \delta_{x}^{2} u_{i}+\alpha u_{i}\left(1-u_{i}\right) .
\end{aligned}
$$

Using the method of lagging to linearize the nonlinear terms, the Crank-Nicolson discretization of equation (10) becomes 


$$
\left(1+\frac{h^{2}}{12} \delta_{x}^{2}\right)\left(\frac{u_{i}^{j+1}-u_{i}^{j}}{k}\right)=\beta \delta_{x}^{2}\left(\frac{u_{i}^{j+1}+u_{i}^{j}}{2}\right)-\frac{\alpha h^{2}}{6}\left(\delta_{x} u_{i}^{j+1}\right)\left(\delta_{x} u_{i}^{j}\right)-\frac{\alpha h^{2}}{12} \delta_{x}^{2}\left(\frac{u_{i}^{j+1}+u_{i}^{j}}{2}\right)+\frac{\alpha h^{2}}{6} u_{i}^{j+1} \cdot \delta_{x}^{2} u_{i}^{j}+\alpha u_{i}^{j+1}\left(1-u_{i}^{j}\right) .
$$

Using the expressions in equation (6) into (11) and simplifying, we get the following tridiagonal system of linear equations:

$$
A_{i} u_{i+1}^{j+1}+B_{i} u_{i}^{j+1}+C_{i} u_{i-1}^{j+1}=D_{i},
$$

where $A_{i}=(2-12 r+\alpha k)+\alpha k\left(u_{i+1}^{j}-u_{i-1}^{j}\right), \quad B_{i}=(20+$ $24 r-26 \alpha k)-4 \alpha k\left(u_{i+1}^{j}-8 u_{i}^{j}+u_{i-1}^{j}\right), C_{i}=(2-12 r+\alpha k)-$ $\alpha k\left(u_{i+1}^{j}-u_{i-1}^{j}\right), \quad D_{i}=(2+12 r-\alpha k)\left(u_{i+1}^{j}+u_{i-1}^{j}\right)+(20-$ $24 r+2 \alpha k) u_{i}^{j}, r=\beta k / h^{2}$.

Equation (12) is called the fourth-order compact Crank-Nicolson scheme for solving the 1D Fisher's equation.

2.2. Stability Analysis. One of the requirements of stability analysis by Von Neumann's method is the linearity of the difference equations [27]. The linear form of equation (10) is given by

$$
\begin{aligned}
\left(1+\frac{h^{2}}{12} \delta_{x}^{2}\right) \frac{\partial u_{i}}{\partial t}= & \beta \delta_{x}^{2} u_{i}-\frac{\alpha h^{2}}{12} \delta_{x}^{2} u_{i}-\frac{\alpha h^{2}}{12} \delta_{x}^{2} u_{i}(1-\text { constant }) \\
& +\alpha u_{i}(1-\text { constant })
\end{aligned}
$$

Replacing $(i h, j k)$ by $(m h, n k)$ in equation (13) to avoid the use of $i$ and $j$ in complex number and simplifying, we arrive at

$$
\begin{aligned}
& (1-6 r+\alpha k) u_{m+1}^{n+1}+(10+12 r+14 \alpha k) u_{m}^{n+1}+(1-6 r+\alpha k) u_{m-1}^{n+1} \\
& =(1+6 r-\alpha k) u_{m+1}^{n}+(10-12 r+2 \alpha k) u_{m}^{n}+(1+6 r-\alpha k) u_{m-1}^{n} .
\end{aligned}
$$

Replacing $u_{m}^{n}$ by $\xi^{n} e^{i m \theta}$ and solving for $\xi$, we get

$$
\begin{aligned}
\xi & =\frac{(1+6 r-\alpha k)\left(e^{i \theta}+e^{-i \theta}\right)+(10-12 r+2 \alpha k)}{(1-6 r+\alpha k)\left(e^{i \theta}+e^{-i \theta}\right)+(10+12 r+14 \alpha k)} \\
& =\frac{2(1+6 r-\alpha k) \cos \theta+(10-12 r+2 \alpha k)}{2(1-6 r+\alpha k) \cos \theta+(10+12 r+14 \alpha k)}, \\
|\xi| & =\left|\frac{2(1+6 r-\alpha k) \cos \theta+(10-12 r+2 \alpha k)}{2(1-6 r+\alpha k) \cos \theta+(10+12 r+14 \alpha k)}\right| \\
& \leq \frac{2|1+6 r-\alpha k||\cos \theta|+|10-12 r+2 \alpha k|}{2|1-6 r+\alpha k||\cos \theta|+|10+12 r+14 \alpha k|} .
\end{aligned}
$$

Since $0 \leq|\cos \theta| \leq 1,|\xi|<1$ for every value of $r>0$. Hence, the proposed method is unconditionally stable.

2.3. Order of the Proposed Method. To discretize the governing equation in equation (1), the first fourth-order compact finite difference method is applied in the space direction, and then, Crank-Nicolson is applied to discretize the derivative involving the temporal variable. Thus, the proposed method in equation (12) has fourth-order accuracy in the space direction and second-order accuracy in the temporal direction. In another word, the order of accuracy of the proposed method is $O\left(h^{4}\right)+O\left(k^{2}\right)$.

2.4. Application of Richardson Extrapolation. One of the advantages of the RE technique is that it increases the accuracy of the approximate solutions of the given differential equation $[15,28]$. It can also be used to accelerate the convergence of the underlying method [22]. The aim is to apply the difference scheme on two consecutive grids and then combine the resulting solutions to obtain higher-order approximate solutions [21].

Consider two consecutive grids $\Omega_{h}^{k}$ coarser and $\Omega_{h / 2}^{k}$ finer and also assume that the following difference equations for solving equation (1) are valid.

$$
\begin{gathered}
U_{h}^{k}=u(x, t)+C h^{4}+r(h, k), \quad\left(x_{i}, t_{j}\right) \in \Omega_{h}^{k}, \\
U_{h / 2}^{k}=u(x, t)+C \frac{h^{4}}{16}+r\left(\frac{h}{2}, k\right),\left(x_{i}, t_{j}\right) \in \Omega_{h / 2}^{k},
\end{gathered}
$$

where $r(h, k)$ and $r(h / 2, k)$ are the remainders of order $O\left(h^{6}, k^{2}\right)$ and $\mathrm{C}$ is a constant independent of $h$ and $k$. To eliminate $C h^{4}$ between the two difference equations, multiply equation (16) by $-1 / 15$ and equation (17) by $16 / 15$ and then combine the resulting difference equations on the coarser gird $\Omega_{h}^{k}$ to get better approximate solution using the following:

$$
u(x, t)=\frac{16 U_{h / 2}^{k}-U_{h}^{k}}{15}+O\left(h^{6}, k^{2}\right), \quad\left(x_{i}, t_{j}\right) \in \Omega_{h}^{k} .
$$

\section{Results, Discussion, and Conclusion}

3.1. Numerical Test Examples. To validate the performance of the proposed method, we considered two numerical examples whose exact solution is available. The pointwise absolute error $\varepsilon$ at $t=T$ is approximated by

$$
\varepsilon=\left|u^{\text {exact }}\left(x_{i}, T\right)-u^{\text {approx }}\left(x_{i}, T\right)\right|, \quad 1 \leq i \leq N-1 .
$$

The $L_{\infty}$ and $L_{2}$ error norms at $t=T$ can also be approximated by

$$
\begin{aligned}
L_{\infty} & =\max _{1 \leq i \leq N-1}\left|u^{\text {exact }}\left(x_{i}, T\right)-u^{\text {approx }}\left(x_{i}, T\right)\right|, \\
L_{2} & =\sqrt{\frac{1}{N} \sum_{i=1}^{N}\left|u^{\text {exact }}\left(x_{i}, T\right)-u^{\text {approx }}\left(x_{i}, T\right)\right|^{2}} .
\end{aligned}
$$

Example 1. Consider the equation (1) $[28,30]$ with $\beta=1$, $\alpha=6$ in $[0,1]$. For this equation, the initial condition and the boundary condition are, respectively, given by 
TABle 1: Comparison between pointwise errors of Example 1 for $\Delta t=0.001$ and $T=0.01$ by the modified Keller Box method [26] and the present method.

\begin{tabular}{|c|c|c|c|c|c|}
\hline Method & $x$ & $N=10$ & $N=20$ & $N=40$ & $N=80$ \\
\hline \multirow{9}{*}{ Present method } & 0.1 & $2.7048 e-05$ & $7.8826 e-06$ & $4.6663 e-06$ & $4.7429 e-06$ \\
\hline & 0.2 & $1.9913 e-06$ & $2.2025 e-06$ & $4.5380 e-06$ & $6.2687 e-06$ \\
\hline & 0.3 & $6.5613 e-06$ & $6.7781 e-07$ & $4.8015 e-06$ & $6.9826 e-06$ \\
\hline & 0.4 & $7.8298 e-06$ & $8.4649 e-07$ & $5.1902 e-06$ & $7.3619 e-06$ \\
\hline & 0.5 & $7.2517 e-06$ & $1.3141 e-06$ & $5.4721 e-06$ & $7.5199 e-06$ \\
\hline & 0.6 & $6.5162 e-06$ & $1.6795 e-06$ & $5.5846 e-06$ & $7.4891 e-06$ \\
\hline & 0.7 & $6.1483 e-06$ & $1.8155 e-06$ & $5.4960 e-06$ & $7.2603 e-06$ \\
\hline & 0.8 & $6.9525 e-06$ & $1.4235 e-06$ & $4.9992 e-06$ & $6.6302 e-06$ \\
\hline & 0.9 & $9.8358 e-06$ & $1.3214 e-07$ & $3.3749 e-06$ & $4.7781 e-06$ \\
\hline \multirow{9}{*}{ Method in [26] } & 0.1 & $3.7 e-05$ & $2.9 e-05$ & $2.7 e-05$ & $2.6 e-05$ \\
\hline & 0.2 & $4.6 e-05$ & $3.5 e-05$ & $3.3 e-05$ & $3.2 e-05$ \\
\hline & 0.3 & $4.4 e-05$ & $3.5 e-05$ & $2.6 e-05$ & $2.6 e-05$ \\
\hline & 0.4 & $3.6 e-05$ & $2.8 e-05$ & $2.6 e-05$ & $2.6 e-05$ \\
\hline & 0.5 & $3.7 e-05$ & $3.1 e-05$ & $2.9 e-05$ & $2.8 e-05$ \\
\hline & 0.6 & $3.4 e-05$ & $2.8 e-05$ & $2.6 e-05$ & $2.5 e-05$ \\
\hline & 0.7 & $3.1 e-05$ & $2.5 e-05$ & $2.4 e-05$ & $2.3 e-05$ \\
\hline & 0.8 & $2.6 e-05$ & $2.1 e-05$ & $2.0 e-05$ & $1.9 e-05$ \\
\hline & 0.9 & $1.8 e-05$ & $1.5 e-05$ & $1.4 e-05$ & $1.3 e-05$ \\
\hline
\end{tabular}

TABle 2: Comparison between $L_{2}$ and $L_{\infty}$ errors norms of Example 1 for $\Delta t=0.0001$ and 0.00005 when $h=0.025$ for different values of $T$.

\begin{tabular}{|c|c|c|c|c|c|c|}
\hline \multirow{2}{*}{$T$} & \multicolumn{3}{|c|}{$\Delta t=0.0001$} & \multicolumn{3}{|c|}{$\Delta t=0.00005$} \\
\hline & $L_{2}$ & $L_{\infty}$ & CPU time (s) & $L_{2}$ & $L_{\infty}$ & CPU time (s) \\
\hline 0.5 & $1.9230 e-05$ & $2.7178 e-05$ & 1.225613 & $3.1680 e-05$ & $4.4632 e-05$ & 2.422009 \\
\hline 1 & $1.8592 e-06$ & $2.4774 e-06$ & 2.652287 & $7.8558 e-07$ & $1.3290 e-06$ & 4.824446 \\
\hline 2 & $1.7754 e-08$ & $2.4029 e-08$ & 4.732449 & $9.1469 e-09$ & $1.2185 e-08$ & 9.554945 \\
\hline 3 & $1.1986 e-10$ & $1.6225 e-10$ & 7.147198 & $6.1787 e-11$ & $8.2297 e-11$ & 14.691121 \\
\hline 4 & $8.2862 e-13$ & $1.1298 e-12$ & 9.560027 & $3.4944 e-13$ & $4.6230 e-13$ & 19.793493 \\
\hline 5 & $8.8105 e-15$ & $1.6431 e-14$ & 12.056541 & $1.7150 e-13$ & $2.4192 e-13$ & 24.023544 \\
\hline
\end{tabular}

$$
\begin{aligned}
& u(x, 0)=\left(1+e^{x}\right)^{-2} ; \quad 0 \leq x \leq 1, \\
& u(0, t)=\left(1+e^{-5 t}\right)^{-2}, \\
& u(1, t)=\left(1+e^{1-5 t}\right)^{-2} ; \quad 0 \leq t \leq T .
\end{aligned}
$$

The exact solution for this equation is given by $u(x, t)=\left(1+\exp \left({ }^{x-5 t}\right)\right)^{-2} ; \quad 0 \leq x \leq 1, t \geq 0$.

Example 2. Consider the equation (1) [29] for $x \in$ $[-0.2,0.8]$ with boundary conditions

$$
\begin{aligned}
& \lim _{x \longrightarrow-\infty} u(x, t)=1, \\
& \lim _{x \rightarrow-\infty} u(x, t)=0 .
\end{aligned}
$$

For this problem, the initial condition is

$$
u(x, 0)=\left[1+\exp \left(\sqrt{\frac{\alpha}{6}} x\right)\right]^{-2}
$$

The exact solution is

$$
u(x, t)=\left[1+\exp \left(\sqrt{\frac{\alpha}{6}} x-\left(\frac{\alpha}{6}\right) 5 t\right)\right]^{-2}
$$

3.2. Discussion. In Table 1, pointwise absolute errors of Example 1 for different values of the spatial step-size with time step-size $\Delta t=0.001$ and $T=0.01$ are tabulated. The displayed results and the comparison of the pointwise absolute errors obtained by the present method and the Keller Box method in [26], show that the present method approximates the exact solution very well. In Table 2, $L_{2}$ and $L_{\infty}$ errors and the computational running time are displayed for two values of time step-size ( $\Delta t=0.0001$ and 0.00005$)$ for different values of $T$ when $h=0.025$. As we increase the value of $T$ from 0.5 to 5 , the errors obtained by $L_{2}$ and $L_{\infty}$ decrease rapidly; however, the computational time increases. The numerical results given in Table 3 clearly show that the present method is a good candidate for solving the 1D Fisher's equation. Furthermore, it can be clearly seen from Tables 2 and 3 that the $L_{2}$ error norm better approximates the exact solution 
TABLE 3: Comparison between $L_{2}$ and $L_{\mathrm{o}}$ errors norms for Example 2 with $\Delta t=0.0001$ and 0.00005 when $h=0.025$ for different values of $T$.

\begin{tabular}{cccccc}
\hline$T$ & \multicolumn{3}{c}{$\Delta t=0.0001$} & & \multicolumn{2}{c}{$\Delta t=0.00005$} \\
& $L_{2}$ & $L_{\infty}$ & CPU time $(\mathrm{s})$ & $L_{2}$ & $L_{\infty}$ \\
\hline 0.1 & $3.8809 e-16$ & $6.6613 e-16$ & 0.597 & $6.5015 e-16$ & $1.1102 e-15$ \\
0.2 & $3.1924 e-16$ & $6.6613 e-16$ & 0.987 & $6.4294 e-16$ & $1.1102 e-15$ \\
0.3 & $3.2715 e-16$ & $6.6613 e-16$ & 1.418 & $6.5659 e-16$ & $1.1102 e-15$ \\
0.4 & $3.2715 e-16$ & $6.6613 e-16$ & 1.459 & $6.5015 e-16$ & $1.1102 e-15$ \\
0.5 & $3.1113 e-16$ & $6.6613 e-16$ & 1.808 & $6.4294 e-16$ & $1.1102 e-15$ \\
\hline
\end{tabular}

TABLe 4: $L_{1}, L_{2}$, and $L_{\mathrm{\infty}}$ errors and computational time for Example 2 at time $T=0.0025$ with the space step-length $h=0.01$.

\begin{tabular}{|c|c|c|c|c|}
\hline \multicolumn{5}{|l|}{ Present method } \\
\hline Time step $(k)$ & $L_{1}$ error & $L_{2}$ error & $L_{\infty}$ error & CPU time (s) \\
\hline $\mathrm{T} / 52$ & $3.5497 e-02$ & $3.5232 e-03$ & $1.5153 e-02$ & 0.340 \\
\hline $\mathrm{T} / 100$ & $2.5420 e-02$ & $1.7665 e-03$ & $7.7685 e-03$ & 0.443 \\
\hline $\mathrm{T} / 200$ & $1.7940 e-02$ & $8.4546 e-04$ & $3.8557 e-03$ & 0.705 \\
\hline $\mathrm{T} / 300$ & $1.4662 e-02$ & $5.4901 e-04$ & $2.5648 e-03$ & 0.958 \\
\hline $\mathrm{T} / 400$ & $1.2720 e-02$ & $4.0708 e-04$ & $1.9218 e-03$ & 1.182 \\
\hline $\mathrm{T} / 500$ & $1.1400 e-02$ & $3.2679 e-04$ & $1.5368 e-03$ & 1.516 \\
\hline $\mathrm{T} / 1000$ & $8.5497 e-03$ & $1.9788 e-04$ & $7.6855 e-04$ & 2.816 \\
\hline $\mathrm{T} / 1200$ & $8.3671 e-03$ & $1.8535 e-04$ & $6.4073 e-04$ & 3.435 \\
\hline $\mathrm{T} / 1400$ & $8.2355 e-03$ & $1.7903 e-04$ & $5.4948 e-04$ & 3.848 \\
\hline $\mathrm{T} / 1600$ & $8.1384 e-03$ & $1.7590 e-04$ & $4.8106 e-04$ & 4.425 \\
\hline \multicolumn{5}{|l|}{ Method in [29] } \\
\hline $\mathrm{T} / 52$ & $2.8968 e-01$ & - & 1.5200 & 0.648 \\
\hline $\mathrm{T} / 100$ & $6.5144 e-01$ & - & $6.9877 e-01$ & 0.719 \\
\hline $\mathrm{T} / 200$ & $3.2980 e-02$ & - & $3.9927 e-01$ & 0.672 \\
\hline $\mathrm{T} / 300$ & $2.0727 e-02$ & - & $2.5613 e-01$ & 0.693 \\
\hline $\mathrm{T} / 400$ & $1.4262 e-02$ & - & $1.7830 e-01$ & 0.708 \\
\hline $\mathrm{T} / 500$ & $1.0268 e-02$ & - & $1.2943 e-01$ & 0.730 \\
\hline $\mathrm{T} / 1000$ & $2.0059 e-03$ & - & $2.6389 e-02$ & 0.904 \\
\hline $\mathrm{T} / 1200$ & $6.2983 e-03$ & - & $9.0013 e-03$ & 1.233 \\
\hline $\mathrm{T} / 1400$ & $4.2509 e-04$ & - & $4.3458 e-03$ & 1.141 \\
\hline $\mathrm{T} / 1600$ & $1.1913 e-03$ & - & $1.3138 e-02$ & 1.366 \\
\hline
\end{tabular}

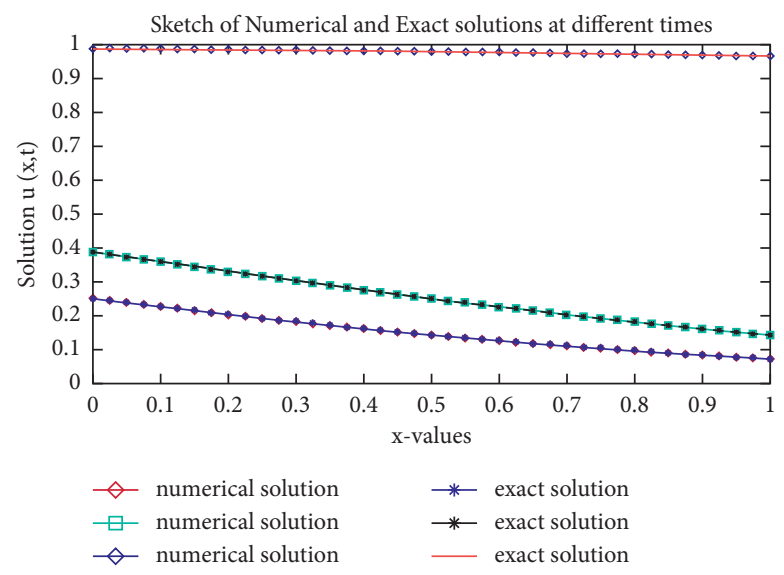

FIgURE 1: Numerical and exact solutions for Example 1 when $h=0.025$ and $\Delta t=0.0001$ for different values of $T$.

than the $L_{\infty}$ error norm. In Table 4 , the numerical results of the present method are compared against the results generated by Agbavon et al. in [30] in terms of $L_{1}$ and $L_{\infty}$ errors, and the results show that our method better approximates the 1D Fisher's equation in equation (1).
In Figures 1 and 2, the numerical solution is sketched against the exact solution for different values of $T$ with $\Delta t=$ 0.0001 and $h=0.025$. From Figures 1 and 2 , one can clearly see that the graphs of the approximate and the exact solutions overlap, showing that there is a good agreement 


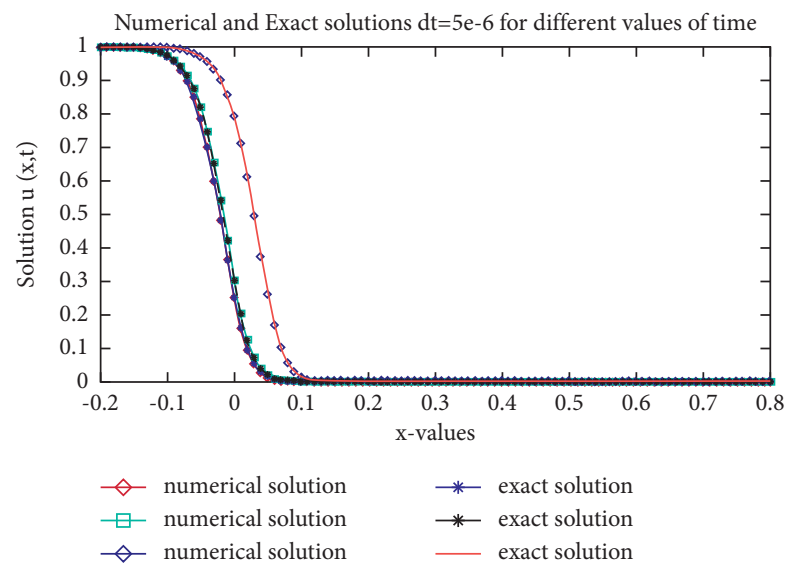

Figure 2: Numerical and exact solutions for Example 2 when $h=0.01$ and $\Delta t=5 e-06$ for some different values of time.

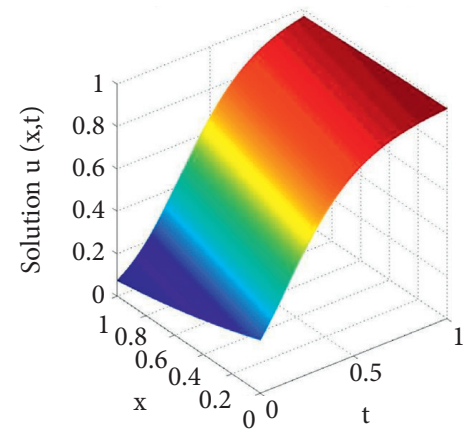

(a)

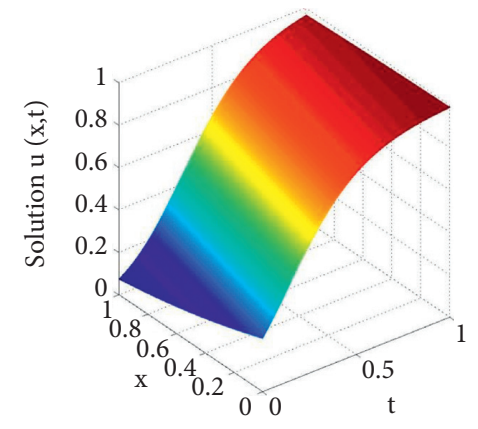

(b)

FIGURE 3: Solution profiles of numerical and exact solutions for Example 1 when $h=0.025$ and $\Delta t=0.0001$ for different values of $T$.

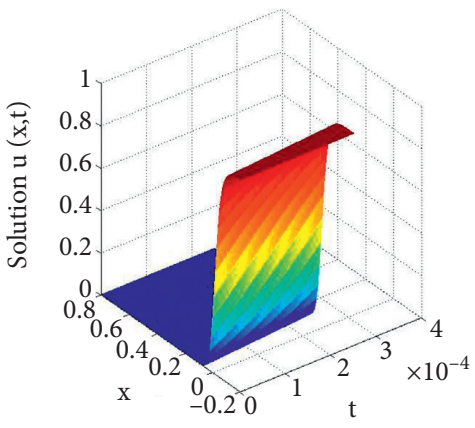

(a)

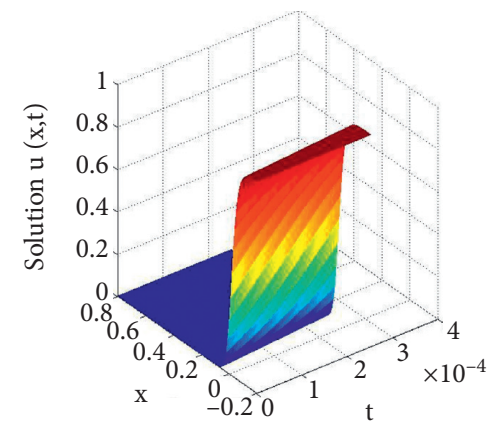

(b)

Figure 4: Numerical and exact solution profiles for Example 2 when $h=0.01$ and $\Delta t=5 e-06$ for some different values of time.

between the approximate and exact solutions. Furthermore, the profiles of the approximate and numerical solutions shown in Figures 3 and 4 also reveal that the present method approximates the exact solution very well.

3.3. Conclusion. In this study, the fourth-order compact Crank-Nicolson scheme combined with Richardson extrapolation for solving the 1D Fisher's equation presented. First, the derivative envolving the space variable is discretized by the fourth-order compact finite difference method. Then, the nonlinear term in the governing equation is linearized by the lagging method and the derivative involving the temporal variable is discretized by the modified Crank-Nicolson scheme. The method is found to be unconditionally stable and fourth-order accurate in the direction of the space variable and second-order accurate in the direction of the temporal variable. When combined with the Richardson extrapolation, the order of the method is improved from fourth to sixth-order accurate in the direction of the space variable. To validate the applicability and efficiency of the proposed method, two numerical examples 
are solved, and the results are presented in tables and figures. From the results displayed in tables and figures, we can conclude that the the presnt method is efficient, accurate, and better approximates the $1 \mathrm{D}$ Fisher's equation than some of the methods presented in the literature.

\section{Data Availability}

The data used to support the findings of this study are included within the article.

\section{Conflicts of Interest}

The authors declare that they have no conflicts of interest.

\section{Acknowledgments}

The authors would like to express their heartfelt thanks to Jimma University for funding this project work.

\section{References}

[1] K. Erwin, Advanced Engineering Mathematics, pp. 535-536, John Wiley and Sons, Ohio state University, NJ, USA, 9th edition, 2006.

[2] R. A. Fisher, "The wave of advance of advantageous genes," Annals of eugenics, vol. 7, no. 4, pp. 355-369, 1937.

[3] R. C. Mittal and R. Jiwari, "Numerical study of Fisher's equation by using differential quadrature method," International Journal Of Information And Systems Sciences, vol. 5, no. 1, pp. 143-160, 2009.

[4] J. Canosa, "On a nonlinear diffusion equation describing population growth," IBM Journal of Research and Development, vol. 17, no. 4, pp. 307-313, 1973.

[5] S. Hasnain and M. Saqib, "Numerical study of one dimensional fishers kpp equation with finite difference schemes," American Journal of Computational Mathematics, vol. 7, no. 1, pp. 70-83, 2017.

[6] J. Gazdag and J. Canosa, "Numerical solution of fisher's equation," Journal of Applied Probability, vol. 11, no. 3, pp. 445-457, 1974.

[7] R. Arnold, K. Showalter, and J. J. Tyson, "Propagation of chemical reactions in space," Journal of Chemical Education, vol. 64 , pp. 740-742, 1987.

[8] H. C. Tuckwell, "Introduction to theoretical neurobiology," Linear Cable Theory and Dendritic Structure, vol. 1, Cambridge University Press, Cambridge, UK.

[9] P. K. Maini, D. L. S. McElwain, and D. Leavesley, "Travelling waves in a wound healing assay," Applied Mathematics Letters, vol. 17, no. 5, pp. 575-580, 2004.

[10] S. K. Aggarwal, "Some numerical experiments on fisher equation," International Communications in Heat and Mass Transfer, vol. 12, no. 4, pp. 417-430, 1985.

[11] S. Tang and R. O. Weber, "Numerical study of fisher's equation by a Petrov-Galerkin finite element method," The Journal of the Australian Mathematical Society. Series B. Applied Mathematics, vol. 33, no. 1, pp. 27-38, 1991.

[12] K. Al-Khaled, "Numerical study of fisher's reaction-diffusion equation by the Sinc collocation method," Journal of Computational and Applied Mathematics, vol. 137, no. 2, pp. 245-255, 2001.

[13] R. C. Mittal and S. Kumar, "Numerical study of fisher's equation by wavelet Galerkin method," International Journal of Computer Mathematics, vol. 83, no. 3, pp. 287-298, 2006.
[14] M. Bastani and D. K. Salkuyeh, "A highly accurate method to solve fisher's equation,” Pramana, vol. 78, no. 3, pp. 335-346, 2012.

[15] F. E. Merga and H. M. Chemeda, "Modified crank-nicolson scheme with richardson extrapolation for one-dimensional heat equation," Iranian Journal of Science and Technology Transaction A-Science, vol. 45, pp. 1725-1734, 2021.

[16] S. K. Lele, "Compact finite difference schemes with spectrallike resolution," Journal of Computational Physics, vol. 103, no. 1, pp. 16-42, 1992.

[17] J. Li and Y. T. Chen, Computational Partial Differential Equations Using MATLAB, CRC Press, Florida, FL, USA, 2008.

[18] Y. M. Wang and H. B. Zhang, "Higher-order compact finite difference method for systems of reaction-diffusion equations," Journal of Computational and Applied Mathematics, vol. 233, no. 2, pp. 502-518, 2009.

[19] G. Sutmann, "Compact finite difference schemes of sixth order for the helmholtz equation," Journal of Computational and Applied Mathematics, vol. 203, no. 1, pp. 15-31, 2007.

[20] S. C. R. Dennis and J. D. Hudson, "Compact h4 finite-difference approximations to operators of Navier-Stokes type," Journal of Computational Physics, vol. 85, no. 2, pp. 390-416, 1989.

[21] W. A. Gaddah, "A higher-order finite-difference approximation with richardson's extrapolation to the energy eigenvalues of the quartic, sextic and octic anharmonic oscillators," European Journal of Physics, vol. 36, no. 3, Article ID 35025, 2015.

[22] M. K. Siraj, G. F. Duressa, and T. A. Bullo, "Fourth-order stable central difference with richardson extrapolation method for second-order self-adjoint singularly perturbed boundary value problems," Journal of the Egyptian Mathematical Society, vol. 27, no. 1, pp. 1-14, 2019.

[23] C. Burg and T. Erwin, "Application of richardson extrapolation to the numerical solution of partial differential equations," Numerical Methods for Partial Differential Equations, vol. 25, no. 4, pp. 810-832, 2009.

[24] V. A. Gordin and E. A. Tsymbalov, "Compact difference scheme for parabolic and Schrödinger-type equations with variable coefficients," Journal of Computational Physics, vol. 375, pp. 1451-1468, 2018.

[25] Z. J. Wang, K. Fidkowski, R. Abgrall et al., "High-order cfd methods: current status and perspective," International Journal for Numerical Methods in Fluids, vol. 72, no. 8, pp. 811-845, 2013.

[26] V. Chandraker, A. Awasthi, and S. Jayaraj, "Implicit numerical techniques for Fisher equation," Journal of Information and Optimization Sciences, vol. 39, no. 1, pp. 1-13, 2018.

[27] G. D. Smith, G. D. Smith, and G. D. S. Smith, "Numerical solution of partial differential equations," in Finite Difference Methods, Oxford University Press, Oxford, UK, 1985.

[28] B. M. Abagero, G. F. Duressa, and H. G. Debela, "Singularly perturbed robin type boundary value problems with discontinuous source term in geophysical fluid dynamics," Iranian Journal of Numerical Analysis and Optimization, vol. 11, no. 2, pp. 351-364, 2021.

[29] G. Arora and V. Joshi, “A computational approach for solution of one dimensional parabolic partial differential equation with application in biological processes," Ain Shams Engineering Journal, vol. 9, no. 4, pp. 1141-1150, 2018.

[30] K. M. Agbavon, A. R. Appadu, and M. Khumalo, "On the numerical solution of Fisher's equation with coefficient of diffusion term much smaller than coefficient of reaction term," Advances in Difference Equations, vol. 2019, no. 1, Article ID 146, 2019. 